\title{
Shock Tube Studies on Recombination Kinetics of Sodium Ion with Electron *
}

\author{
WANG Su(王苏), CUI Ji-Ping(崔季平), HE Yu-Zhong(何宇中), FAN Bing-Cheng(范秉诚), WANG Jing(王静) \\ Laboratory of High Temperature Gas Dynamics, Institute of Mechanics, Chinese Academy of Sciences, Beijing 100080
}

(Received 2 June 2000)

\begin{abstract}
The ionization kinetics of sodium diluted in argon is studied in a shock tube, in which the test gas mixture is ionized by a reflected shock wave and subsequently quenched by a strong rarefaction wave. A Langmuir electrostatic probe is used to monitor the variation of the ion number density at the reflection shock wave region. The working state of the probe is in the near free fall region and a correction for reduction of the probe current due to elastic scattering in the probe sheath is introduced. At the temperature range of 800 to $2600 \mathrm{~K}$ and in the ambience of argon gas, the three-body recombination rate coefficient of the sodium ion with electron is determined: $3.43 \times 10^{-14} \mathrm{~T}^{-3.77} \mathrm{~cm}^{6} \cdot \mathrm{s}^{-1}$.
\end{abstract}

PACS: 82.20.Pm, 34.50.Fa, 07.35.+k

Alkali metal atoms have the lowest ionization potentials in all families in the elementary periodic table. Adding a small amount of alkali metal substance will change the ionizing state of a gas. The previous studies on recombination rates of alkali metal ions with electrons were performed in a flame by use of the diagnostic techniques including electrostatic probe, ${ }^{1,2}$ microwave cavity resonance, ${ }^{3}$ radio frequency resonance, ${ }^{4}$ ion mass spectroscopy, ${ }^{5}$ atom spectroscopy ${ }^{6}$ and planar laser induced fluorescence. ${ }^{7}$ However, all these experiments were restricted within the narrow limits of the flame temperature of 2000 $2500 \mathrm{~K}$. In addition, complicated composition and chemical reactions in the flame interfere with the determination of the recombination rate coefficient.

In this work, a new shock tube technique is applied to determine directly the three-body recombination rate coefficient of the sodium ion with electron in a wider test temperature range, where the ionized gas is obtained by a reflected shock wave and subsequently cooled down by a strong rarefaction wave reflected at the end wall of the high pressure section of the shock tube. As the cooling speed of the strong rarefaction wave is as high as $10^{6} \mathrm{~K} / \mathrm{s}$, the temperature in the reflection shock wave region could decreases from 3000 to $600 \mathrm{~K}$ within the experimental time of about $3 \mathrm{~ms}$ and a non-equilibrium ionization state is attained at the later stage of the process. Furthermore, the whole process is in an unadulterated environment.

The experiments are carried out in a single pulse shock tube. ${ }^{8}$ The apparatus diagram is shown in Fig. 1. Sodium amide is chosen as the source substance introducing the sodium ion into the experimental system. Sodium amide in solid state is ground into powder under the protection of benzene, which is volatilized in the shock tube and excluded out by evacuation before every experimental run. The diameters of grains of sodium amide in the suspension of benzene are checked to be not more than $20 \mu \mathrm{m}$. The shock tube is working at the fixed initial temperature
$T_{1}=343 \mathrm{~K}$ and pressure $P_{1}=4 \mathrm{kPa}$, but with shock Mach number ranging from $M_{\mathrm{s}}=3.26$ to 3.47 .

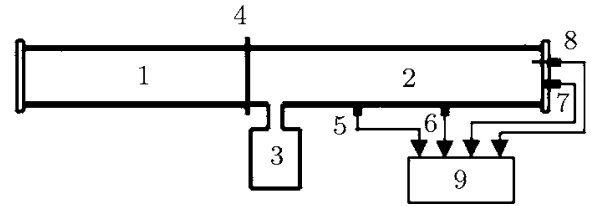

Fig. 1. Apparatus diagram of the single pulse shock tube: (1) high pressure section; (2) low pressure section; (3) dump tank; (4) diaphragm; (5-7) piezo-electric transducer; (8) Langmuir electrostatic probe; (9) transient converter.

A piezo-electric transducer is used to monitor the pressure evolution of the whole process. The rarefaction wave cooling stage is treated as an adiabatic process. The inert gas Ar is chosen as a diluent in the present experiment so that the density and temperature of the test gas could simply be calculated from the pressure by use of the adiabatic relations

$$
\frac{\rho}{\rho_{50}}=\left(\frac{P}{P_{50}}\right)^{1 / \gamma}, \quad \frac{T}{T_{50}}=\left(\frac{P}{P_{50}}\right)^{(\gamma-1) / \gamma},
$$

where $\gamma=5 / 3$ is the adiabatic index of argon and the subscript 50 refers to the corresponding initial state just behind the reflected shock wave.

In the present experiment, the number density of the sodium ion, $\mathrm{N}_{\mathrm{Na}^{+}}$, is measured by a cylindrical Langmuir electrostatic probe with a good spatial resolution and sensitivity, as is widely used now. ${ }^{9}$ The probe, with a radius $r_{\mathrm{p}}=0.01 \mathrm{~cm}$ and a length $l_{\mathrm{p}}=1.3 \mathrm{~cm}$, is biased negatively at $V_{\mathrm{p}}=-9 \mathrm{~V}$. The Debye length of the ionized gas in our typical case is approximately $\lambda_{\mathrm{D}} \approx 10^{-3} \mathrm{~cm}$, and the mean free path of the sodium ion in the ambience of argon gas is estimated as $\lambda_{\mathrm{i}} \approx 10^{-4} \mathrm{~cm}$. Since $\lambda_{\mathrm{D}}>\lambda_{\mathrm{i}}$, the probe works in the near free fall. Therefore the influence of the collision of the ion with neutral atoms in the probe sheath on the probe collecting current must be taken into account. According to the theories given by Schulz and Brown ${ }^{10}$ and Jakubowski, ${ }^{11}$ the correction 
factor for reduction of the probe current due to elastic scattering is

$$
\begin{gathered}
\alpha= \begin{cases}\frac{3-2 \exp \left(-\lambda_{\mathrm{D}} / \lambda_{\mathrm{i}}\right)}{1+2\left(\lambda_{\mathrm{D}} / \lambda_{\mathrm{i}}\right)}, & \lambda_{\mathrm{D}} / \lambda_{i}<1 \\
\frac{3-\exp \left(-\lambda_{\mathrm{D}} / \lambda_{\mathrm{i}}\right)}{2\left(1+\lambda_{\mathrm{D}} / \lambda_{\mathrm{i}}\right)}, & \lambda_{\mathrm{D}} / \lambda_{i} \geq 1\end{cases} \\
\lambda_{\mathrm{D}}=\left(\frac{k T}{4 \pi e^{2} N_{\mathrm{Na}^{+}}}\right)^{1 / 2}, \quad \text { and } \quad \lambda_{\mathrm{i}}=\left(N_{\mathrm{Ar}} \sigma_{0}\right)^{-1}
\end{gathered}
$$

where $\lambda_{\mathrm{D}} / \lambda_{\mathrm{i}}$ is the average collision number in the sheath, $\sigma_{0}$ the total cross section for the elastic scattering between the sodium ion and argon atoms and $N_{\text {Ar }}$ the number density of argon atoms.

As $r_{\mathrm{p}} / \lambda_{\mathrm{D}}$ is quite large, scattering with small deflection angles due to collisions will not cause the falling ions to fly off from the probe. As $r_{\mathrm{p}} / \lambda_{\mathrm{D}} \approx 10$ in the present experiment, only the collisions that lead to large deflection angles should be considered. Then we establish the interaction potential for elastic collisions of the sodium ion with argon atoms by using the repulsion potential part of the Buckingham potential and the induction potential between the ion and non-polar molecule. The result is given by

$$
\begin{aligned}
\varphi(r)= & 4.28 \times 10^{-3} \exp \left[14.25\left(1-\frac{r}{3.56}\right)\right] \\
& -1.02 \times 10^{-2}\left(\frac{5.95}{r}\right)^{4}
\end{aligned}
$$

with distance $r$ in angstrom and interaction potential $\varphi$ in $\mathrm{eV}$, where the parameters of the Buckingham potential are adopted from Ref. 12 and the induction potential coefficient is determined by use of the value of the induction potential at the distance equal to the kinetic collision diameter calculated by Margenau. ${ }^{13}$ The so-called close collision cross section $\sigma_{\mathrm{c}}$, which leads to deflection angles greater than $\pi / 2$, is obtained as the effective collision cross section through integrating over the collision trajectory so as to calculate the average collision number in the probe sheath. Finally the average collision number and the sodium ion number density can be obtained as

$$
\begin{aligned}
& \frac{\lambda_{\mathrm{D}}}{\lambda_{\mathrm{i}}}=6.9 \sigma_{\mathrm{c}} T^{1 / 2} N_{\mathrm{Ar}} N_{\mathrm{Na}^{+}}^{-1 / 2} \\
& N_{\mathrm{Na}^{+}}=1.01 \times 10^{17} \frac{I_{\mathrm{i}} T^{-1 / 2}}{\alpha},
\end{aligned}
$$

where $I_{\mathrm{i}}$ is the probe current measured in ampere.

The cold boundary layer of the probe may grow unsteadily as a result of interaction of the probe with a sequence of vortices with length scale of the order of $r_{\mathrm{p}}$. Using Lin's boundary layer theory of oscillating flow, ${ }^{14}$ the maximum thickness of the boundary layer growing unsteadily is estimated as $\delta_{\max } \approx 10^{-4} \mathrm{~cm}$. For $\delta_{\max } \ll \lambda_{\mathrm{D}}$, the effect of cold boundary layer is negligible.
The kinetic equation of the recombination process of the sodium ion with electron is given by

$$
\begin{aligned}
k_{\mathrm{r}} N_{\mathrm{Na}}+N_{\mathrm{Ar}}= & \frac{\mathrm{d} \ln \rho}{\mathrm{d} t}-\frac{\mathrm{d} \ln N_{\mathrm{Na}^{+}}}{\mathrm{d} t} \\
& +\frac{f\left(T, T_{\mathrm{S}}\right)}{N_{\mathrm{Na}^{+}}}\left(\frac{\rho}{\rho_{\mathrm{S}}}\right)^{2} \frac{\mathrm{d} N_{\mathrm{Na}}+\mathrm{S}}{\mathrm{d} t} \\
f\left(T, T_{\mathrm{S}}\right)= & \left(\frac{T}{T_{\mathrm{S}}}\right)^{1 / 2} \exp \left[-\frac{\theta^{*}}{T}\left(1-\frac{T}{T_{\mathrm{S}}}\right)\right],
\end{aligned}
$$

where $\theta^{*}=2.42 \times 10^{4} \mathrm{~K}$ is the characteristic temperature for the first excitation of the sodium atom and the subscript $\mathrm{S}$ denotes an average over the heating stage of the reflected shock wave.

At the early phase of the cooling process of the rarefaction wave, the second and third terms on the righthand side of Eq. (7) are almost the same because in this phase the ionization and recombination processes are nearly in equilibrium. The data adopted in this phase are not available for determining the recombination rate coefficient $k_{\mathrm{r}}$. As the non-equilibrium effects become obvious, the data adopted in the later phase, $t-t_{\mathrm{M}}>0.5 \mathrm{~ms}$, therefore, are employed in the data interpretation. The $t_{\mathrm{M}}$ is the point that the rarefaction wave starts cooling down. Under this condition, the contribution of the third term on the right-hand side of Eq. (7) is negligible.

Combining Eqs. (2), (5) and (6) with Eq. (7) and using the measured temperature and density, the variation of the three-body recombination rate coefficient with temperature is obtained, as shown in Fig. 2. The different symbols on the graph correspond to different running shock Mach number. The observed rate coefficient is expressed by the least-squares analysis of all the data points in the seven runs as

$$
k_{\mathrm{r}}=3.43 \times 10^{-14} T^{-3.77} \mathrm{~cm}^{6} \cdot \mathrm{s}^{-1}
$$

at the temperature range of $800<T<2600 \mathrm{~K}$.

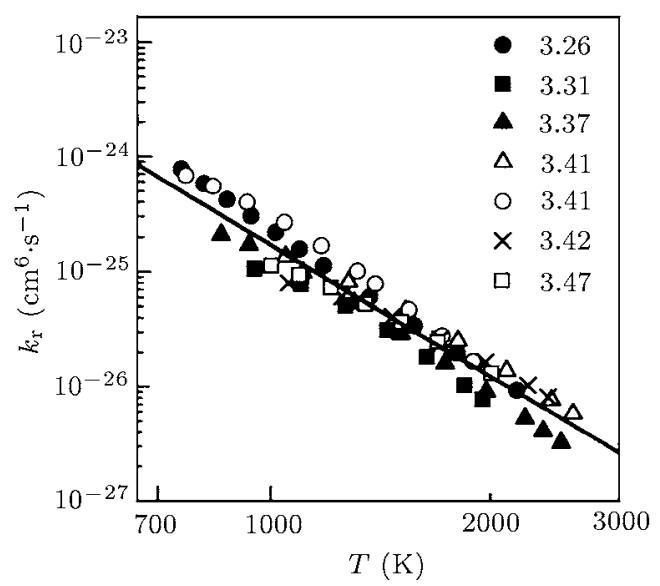

Fig. 2. Variation of the three-body recombination rate coefficient of the sodium ion and electron with temperature. 


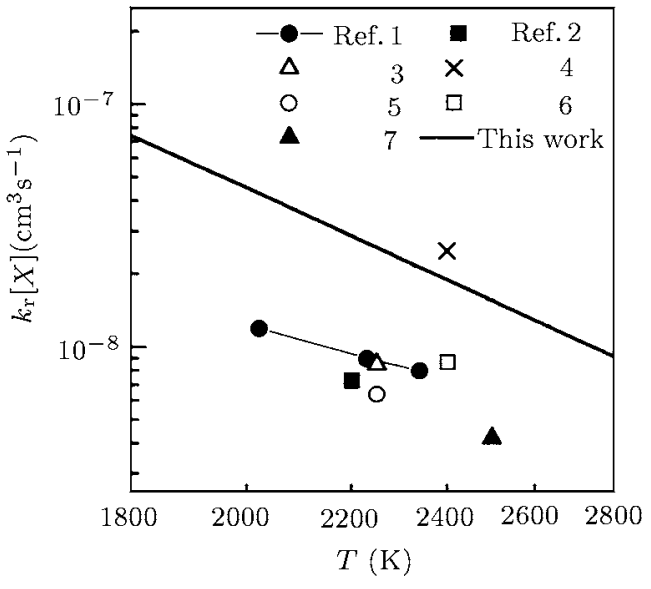

Fig. 3. Comparison of the two-body recombination rate coefficient at the ambient pressure of $1 \mathrm{~atm}$.

If an electron acted as the third body in the collision, Zeldovich ${ }^{15}$ deduced the three-body ion-electron recombination rate coefficient as

$$
k_{\mathrm{re}}=1.71 \times 10^{-8} T^{-4.5} \mathrm{~cm}^{6} \cdot \mathrm{s}^{-1}
$$

by taking Thomson radius $2 e^{2} /(3 k T)$ as the characteristic range of interaction of ions. The collision efficiency of the electron as the third body is 100 times greater than that of the argon atom. In the present work, however, the equilibrium degree of ionization of the sodium atom is approximately $10^{-4}$ and $N_{\mathrm{e}} / N_{\mathrm{Ar}} \approx 10^{-6}$. Considering both concentration and collision efficiency, the third body in the recombination process should be the diluent argon atom rather than the electron. As the interaction potential between the electron and argon atom is the induction potential in the form $\varphi_{\text {ind }}=C / r^{4}$, the characteristic range of the interaction is $[2 C /(3 k T)]^{1 / 4}$ instead of the Thomson radius. A procedure similar to that employed by Zeldovich yields the temperature dependence of the ion-electron recombination coefficient as $k_{\text {ra }} \propto T^{-3.75}$ under the condition that an atom acts as the third body in the collision process. This result is nearly identical with the temperature dependence $T^{-3.77}$ obtained in the present experiment.

The comparison of the two-body recombination rate coefficient of the sodium ion with electron $k_{\mathrm{r}}[X]$ between the present work and other works ${ }^{1-7}$ at the ambient pressure of $1 \mathrm{~atm}$ is shown in Fig. 3, wherein
$X$ expresses the third body of collision. $X$ is the argon atom in our case, but in a flame experiment it denotes all the atoms and molecules existing there. At the flame temperature range, the result of the present work is of the same order as those of the flame experiments. However, the exponent of temperature dependence is more accurately determined, because a wider temperature range is reached in our work.

In summary, applying a new shock tube method together with the electrostatic probe technique, under the condition of the argon atom acting as the third body in the collision process, the three-body recombination rate coefficient of the sodium ion with electron is determined as $k_{\mathrm{r}}=3.43 \times 10^{-14} T^{-3.77} \mathrm{~cm}^{6} \cdot \mathrm{s}^{-1}$ at the temperature range of $800<T<2600 \mathrm{~K}$. The influence of the collision of ion with neutral atoms in the probe sheath is corrected. When an atom acts as the third body, temperature dependence of the three-body recombination rate coefficient is discussed. The result of the present work is of the same order as those of the flame experiments at the flame temperature range, but covers a wide range of temperature.

\section{References}

[1] Anthony E J et al. 1971 J. Chem. Soc. Faraday Trans. I 701067

[2] Kelly R, Padley P J 1969 Trans. Faraday Soc. 65355 Kelly R, Padley P J 1972 Proc. R. Soc. A 327345

[3] Jensen D E and Padley P J 1967 Proc. of 11th Int. Symp. on Combustion (Pittsburgh: Combustion Institute) p 351

[4] Borgers A J et al. 1981 J. Chem. Soc. Faraday Trans. I 111075

[5] Hayhurst A N and Sugden T M 1966 Proc. R. Soc. A 293 36

[6] Hollander T et al. 1963 J. Chem. Phys. 392558

[7] Barker P and Rubinsztein-Dunlop H 1997 Spectrochimica Acta, B 52459

[8] Cui J P et al. 1999 Proc. of 22nd Int. Symp. on Shock Waves (London, UK: Imperial College) p 69

[9] Passoth E et al. 1997 J. Phys. D: Appl. Phys. 301763

[10] Schulz G J and Brown S C 1955 Phys. Rev. 981964

[11] Jakubowski A K 1972 AIAA J. 8988

[12] Mason E A and Rice W E 1954 J. Chem. Phys. 22843

[13] Margenau H 1941 Phil. Sci. 8603

[14] Lin C C 1957 Proc. of 9th Intern. Cong. on Applied Mechanics (Brussels) vol. 4, p 155

[15] Zeldovich Y B and Raizer Y P 1966 Physics of Shock Waves and High Temperature Hydrodynamic Phenomena (New York and London: Academic Press) chap 6 